\title{
VALORACION DEL BOSQUE SECO ALGARROBAL POR MEDIO DE UN PROGRAMA DE EDUCACION AMBIENTAL
}

\section{RECOGNIZING OF DRY FORETS ALGARROBAL BY INSTRUCTIVE THE PROGRAM OF ENVIROMENTAL EDUCATION}

\section{RESUMEN}

\author{
Alfredo Augusto Alzamora Arévalo ${ }^{a}$
}

El presente estudio analiza la importancia de un programa de educación ambiental y observa cómo influye en la valoración del ecosistema del bosque seco algarrobal por parte de los estudiantes del primer grado de educación secundaria de la IEFAP "Samuel Ordóñez V." y "C.N. San Miguel" de Piura. El tipo de investigación es explicativa, utilizando el método cuantitativo-cualitativo, con un diseño cuasi-experimental para lo cual pusimos en práctica un Pre Test y Post Test, para medir las capacidades específicas del área de Ciencia Tecnología y Ambiente referida al contenido diversificado del bosque seco. El programa de educación ambiental tuvo como escenario los bosques de áreas naturales reconocidas por organismos de la región, cuyo ámbito de estudio se situó en el Parque Municipal Kurt Beer (Piura), Comunidad Campesina San Francisco de Locuto (Tambogrande) y Comunidad Campesina Ignacio Távara (Chulucanas) por medio de la técnica de visitas instructivas. Los resultados -analizados mediante técnicas de estadística descriptiva e inferencial - demuestran con valores cuantitativos y cualitativos que es favorable el aprendizaje y formación integral de los estudiantes; llegando a la conclusión de que el programa de educación ambiental por medio de las visitas instructivas es significativo para la valoración del ecosistema del bosque seco en relación con las capacidades, destrezas y actitudes que deben aprender los estudiantes de la región Piura, para la conservación y uso sostenible de este recurso.

Palabras claves: educación ambiental, valoración, bosque seco, Piura.

\section{ABSTRACT}

The present research analyzes the importance of a program of environmental education and how it influences on recognizing the real meaning of the ecosystem of dry forest "algarrobal", being part of the development of this research the students of first grade of secondary education at IEFAP " Samuel Ordóñez V" and "C.N. San Miguel" of Piura. The investigation type is explanatory using the quantitative and qualitative method, with a quasi-experimental design so a pre environment area referred to the diversified topic of the dry forest was applied. The program of environmental education aimed at the forests of natural areas recognized by organisms of the region, of which environment study was located at the Municipal Park called Kurt Beer in Piura, Rural Community San Francisco of Locuto in Tambogrande and Rural Community Ignacio Távara in Chulucanas, used the technique of instructive visits. The results were analized by means of descriptive statistics and inferential techniques demonstrate with quantitative and qualitative results where the learning and the students integral formation are favourable. Thus, it is concluded that the program of environmental education by instructive visits is significant for the valuation of the ecosystem of the forest with close relationship to the capacities, skills and attitudes that students from Piura should learn to keep and use this natural resource suitably.

Key words: environmental education, valuation, dry forest, Piura.

\section{INTRODUCCION}

La presente investigación aborda un contenido transversal del Diseño Curricular Nacional como es la educación ambiental y orientado al ecosistema típico de la región Piura como es el bosque seco algarrobal, como característica del currículo diversificado y al mismo tiempo utilizado como recurso de enseñanza aprendizaje convirtiéndose en una aula natural para hacer educación ambiental con los estudiantes del nivel secundaria

El currículo del área de Ciencia Tecnología y Ambiente (CTA) del primer grado de secundaria de la provincia y región Piura no contempla en sus contenidos lo referente al bosque seco. Como consecuencia los educandos no reconocen la valoración que tiene este ecosistema y por ello es que a partir de un Programa de Educación Ambiental vía los viajes de estudios a los

\footnotetext{
${ }^{a}$ Docente de la IE. Samuel Ordonez y C.N San Miguel de Piura
}

bosque de algarrobo observen la riqueza e importancia que tiene para la región norte (Piura), el Perú y el mundo este recurso natural y a partir de ello valorarlo por medio de ciertas capacidades y destrezas. Son objetivos de nuestra investigación determinar las capacidades y actitudes de valoración al bosque seco por parte de los estudiantes después de participar en un Programa de Educación Ambiental (PEA),Identificar el nivel de valoración hacia el bosque seco, que tiene los estudiantes y Proponer un PEA desde el área de CTA que ayude a la valoración del bosque seco.

El tema de educación ambiental, si bien es cierto es un tema transversal del Diseño Curricular Nacional en tanto existe una honda preocupación por el entorno, no obstante éste no se visualiza en actitudes positivas hacia los recursos naturales por lo que concebimos que a través del campo educativo debemos abordar con mayor énfasis la importancia que tienen estos recursos si son 
usados de manera racional. Por ello nuestra propuesta apunta a tratar -desde el área curricular de Ciencia Tecnología y Ambiente del primer grado de secundariaesta perspectiva de ver la educación ambiental en el campo mismo de los hechos.

La naturaleza de la presente investigación tiene, pues, un carácter de propuesta, para ser utilizada por los docentes de los niveles de educación primaria y secundaria porque contiene materiales organizados en una serie de actividades significativas que el docente puede aprovechar adaptándolas a su necesidad e interés, de tal manera que las visitas de estudio sean para aprovechar lo que tenemos más cerca los piuranos, como es nuestro bosque seco de algarrobos, que puede ser motivador cuando se tenga que salir al campo, y qué mejor si se le da un carácter integrador de áreas del conocimiento llámese Matemática, Comunicación, Arte, Ciencias Sociales; pero sobretodo de carácter actitudinal, que el estudiante aprenda a valorar lo que tiene en materia de recursos naturales, para así poderlos defender, proteger, usar de manera sostenible y sentirse orgulloso de lo que tiene.

\section{MATERIALES Y METODOS}

En estas actividades se utiliza una metodología activa y participativa, que trata al estudiante que visita el área de estudio como el principal componente del proceso educativo y procura un aprendizaje significativo, donde se relacione lo vivencial y experimental con lo que se aprende por medio de la investigación científica. Como podemos apreciar, las visitas instructivas, planificadas ahondan el conocimiento de la realidad de estudio en los educandos. Los educandos llegan al área de estudio, reciben las recomendaciones para el trabajo en el bosque, se inicia con una dinámica para afinar los sentidos y conocer la naturaleza del bosque, luego los equipos de investigación se organizan y se dirigen según el sector de trabajo. Se inicia la caminata por el bosque, donde los educandos irán descubriendo sus secretos.

Los contenidos del programa de educación ambiental están orientados hacia un enfoque integral, principalmente hacia las ciencias ambientales, mediante el método científico. Con este método los educandos observan, discuten, manipulan e infieren sobre la naturaleza, con la guía y orientación del docente. El niño infiere en este aprendizaje todos estos aspectos mencionados, el educador es el orientador-guía que, después, viene a reafirmar conceptos y conocimientos. Se trata de que los estudiantes mediante la observación e investigación lleguen al conocimiento por ellos mismos. Hay que enfatizar que esta manera de aprender es novedosa e innovadora del proceso formal de aprendizaje. Es por ello que esta forma de aprender en el programa de educación ambiental guía al educando a ser más inquisitivo, más capaz de aprender por sí mismo y más inclinado a no aceptar los hechos sin pensar.

Un día de campo corresponde a varias horas de trabajo, con caminatas por el bosque; los educandos utilizan materiales que ayudan a una mejor observación de algunas especies: lupas, redes, vasos plásticos, cintas métricas, guías de campo, redes acuáticas, termómetros, cámaras de video, fotográfica, botiquín, etc. Asimismo, cada educando cuenta con su libreta de apuntes, lápices de colores, cartulina, regla, plumones, papelotes etc.Los informes preliminares son expuestos a manera de talleres a la sombra de los árboles y los informes finales se presentan a manera de simposio en el aula.

El trabajo se realizo teniendo en cuenta la aplicación de la variable independiente Programa de educación Ambiental con lo estudiantes del primer grado de secundaria .El método cuantitativo consistió en aplicar un pretest con 14 items e indicadores con una escala vigesimal acerca de la valoración del bosque seco que tienen los estudiantes, obteniendo al término de la de la prueba cuatro resultados: una calificación por cada una de las tres capacidades del area de Ciencia tecnología y Ambiente y un promedio de las tres respectivas. Posteriormente se aplicó el Programa de Educación Ambiental por medio de la técnica de visitas instructivas al bosque seco algarrobal de diversas áreas de la región tales como "Parque Ecologico Municipal Kurt Beer de Piura, El bosque de la Comunidad Campesina "San Francisco de Locuto" de Tambogrande y el Bosque de la Comunidad Campesina "Ignacio Távara" de Chulucanas. Para la puesta en práctica del Programa de Educación Ambiental, los estudiantes han utilizado estrategias correspondientes al uso y aplicación del método científico.

\section{RESULTADOS}

Al concluir dicho programa se aplicó un test de salida que nos permitió determinar de manera cuantitativa en base a los valores numéricos la valoración vigesimal del contenido de bosque seco, tanto en el Pretest y post test. Con los resultados numéricos de los test correspondientes se hizo una valoración cualitativa comparando tres escalas:

- Esta iniciando el aprendizaje.(C)

- Esta en proceso de aprendizaje (B)

- Logró aprender(A).

Ello nos permitió hacer la evaluación correspondiente en la muestra de estudio acompañado de graficas estadísticas y sus respectivas interpretaciones.

\section{DISCUSION.}

Los hallazgos obtenidos en esta muestra poblacional nos demuestra que existe una influencia notoria y positiva del Programa de Educación Ambiental en la valoración del bosque seco tal como lo confirman los valores de la estadística descriptiva e inferencial del Pre Test y Post Test. Esto indica que existe una significativa diferencia en forma cualitativa y cuantitativa entre el Pre Test y el Post test, Al cruzar los resultados de ambas pruebas vemos la influencia positiva puesto que los resultados se invierten de manera porcentual. (ver cuadro 1)

Nuestra experiencia reporta el desarrollo de actividades experimentales dentro de la misma naturaleza a nivel de educación secundaria. Por esta razón las visitas instructivas son una estrategia que involucra la 
posibilidad no sólo de aprender Ciencia, Tecnología y Ambiente sino también otras áreas por ser un contenido transversal de mucha importancia para toda la población.

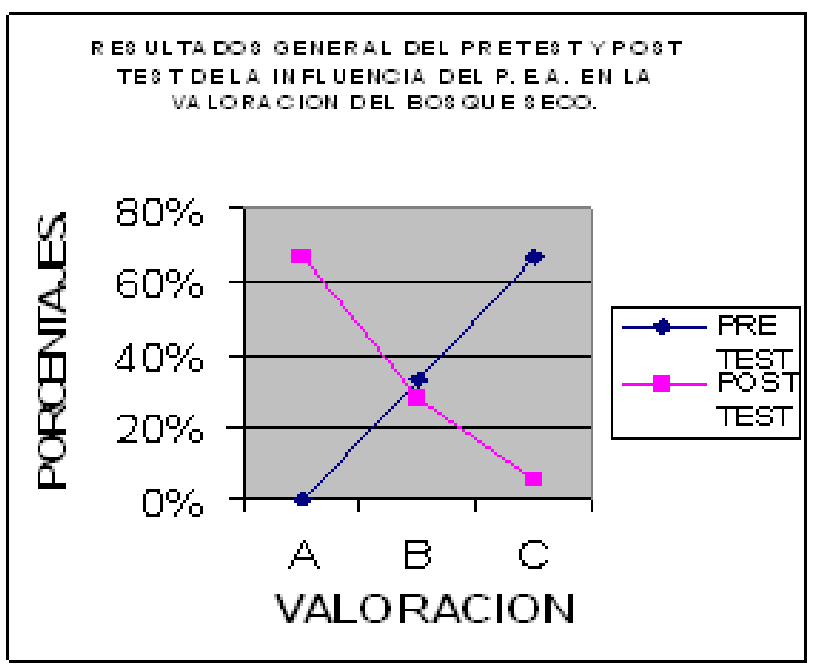

Cuadro 1. resultado comparativo entre el pretest y post test para la valoración del bosque seco por medio de un PEA

\section{CONCLUSIONES.}

- La influencia del Programa de Educación Ambiental es cuantitativamente y cualitativamente significativo en cuanto a la valoración del bosque seco algarrobal .

- Los estudiantes logran desarrollar destrezas como: describe, infiere, reconoce, clasifica, discrimina, formula hipótesis a las diversas situaciones problemáticas del bosque seco; así como opina, argumenta, analiza y plantea alternativas a los problemas y amenazas de este ecosistema.

- El bosque seco algarrobal es un laboratorio natural que nos permite desarrollar actividades experimentales que viabilizan el aprendizaje de las ciencias, de la educación ambiental y una mayor actitud valorativa por el ambiente.

- Se ha logrado aplicar fundamentos de Diseño Curricular Nacional como la educación ambiental como tema transversal y del bosque seco como contenido diversificado de la región Piura

\section{REFERENCIAS BIBLIOGRAFICAS.}

[1]. BERNEX, Nicole. Nosotros y los Andes. Ambiente y Educación. Lima: Ministerio Educación-COSUDE;1997

[2]. BRACK, Antonio; PLENGE Heinz. Perú Maravilloso.Lima: Editorial EPENSA;2002

[3]. BRACK, Antonio; Mendiola, Cecilia.Ecología del Perú. Lima: Editorial Bruño; 2000.

[4]. DIRECCION REGIONAL DE EDUCACION. PIURA. Lineamientos para la Diversificación Curricular en la Región. Piura: S/e ; 2004

[5]. FROTA PESSOA, Oswaldo .Principios Básicos para la enseñanza de la Biología. Washington D.C.: OEA.; 1967

[6]. GONZALES, Elia.. Manual Práctico de Educación Ambiental. Loja: Ediciones Fundación Arco Iris;1986

[7]. MINISTERIO DE EDUCACION . Diseño Curricular Nacional de Educación

[8]. Lima: MINEDU;2005.

[9]. VELÁSQUEZ DE CASTRO, Federico. Educación Ambiental. Madrid: Ediciones NARCEA;1995

[10]. WUST, Walter. Ecología del Perú. Lima: Editora Nacional. Diario Expreso;2000

E-mail: alzamora3a@yahoo.com alzamora3a@hotmail.com 